\title{
Experimental investigation of moist-air transport through natural materials porous media
}

\author{
I. Conte \& X. Peng \\ Laboratory of Phase-Change and Interfacial Transport Phenomena, \\ Tsinghua University, China
}

\begin{abstract}
Experimental investigations were conducted to characterize the moisture absorption of two different natural materials (coconut fibres and groundnut coquets). Two experimental samples were made: one with groundnut coquets (composed by void space and grain) and the other sample was made with coconut fibres (composed by void space and fibres). The samples were multi-layered sandwiches bounded on the top by ice while the bottom surface was exposed to the vapour with temperature close to $100^{\circ} \mathrm{C}$. The percentage of water accumulation due to vapour absorption was calculated for each layer after a pre-set time of experiment $(1 \mathrm{~h}, 2 \mathrm{~h}, \ldots)$. The water content within both the coconut fibres and groundnut coquet increased with time. For the groundnut coquet sample the water was transferred mainly by diffusion through the solid; and in the coconut fibres sample with higher porosity, the water transferred by diffusion through void space was the most important. Since water is a better thermal medium, the thermal conductivity of the soaked insulation would increase and the insulating value of the material would be destroyed.
\end{abstract}

Keywords: natural material, air and moisture transport, porous media.

\section{Introduction}

Many natural materials (such as cork, coconut fibre...) are used in thermal insulation thanks to their relatively low thermal conductivity, low cost, harmless behaviour to the environment and human health. These materials are commonly used for insulation of low temperatures systems such as coldstore and living building because of their bad behaviour under high temperatures. However, one 
main disadvantage of using most of natural materials in thermal insulation is the low resistance to moisture absorption. When heat transfer occurs through a separating wall because of the temperature difference, water vapour diffusion also occurs due to the pressure difference across the wall. As the temperature within the insulation decreases, the moisture already existing in the insulation may condense. Thermal insulation carrying cold fluids suffers thermal degradation due to condensation of water. The value of the water vapour transfer coefficient of the insulation materials is as important as that of the heat transfer coefficient. Many theoretical and experimental investigations of thermal conductivity and moisture absorption and condensation in porous media have been performed, with the aim of applying the results to thermal insulation and other industrial applications. Conte et al. [1] presented experimental investigations of the thermal conductivity of two natural low cost materials, coconut fiber and groundnut coquet. For the purpose of comparison, investigations were done for coconut fiber, groundnut coquet and aluminum silicate fiber as a standard insulation material. Their results showed that the thermal conductivities of both coconut fiber and groundnut coquet (with an average value of $0.08 \mathrm{~W} / \mathrm{m}$. $\mathrm{k}$ ) increase with temperature as well as the thermal conductivity of the aluminum silicate fibers. The measured thermal conductivities showed that the coconut fibers and groundnut coquets may be used as thermal insulation for the tested temperature range. Fan et al. [2] reported an experimental investigation of the temperature and water content distribution within porous fibrous battings sandwiched by an inner and outer layer of thin covering fabrics using a novel sweating, guarded hot plate. They found that most of the change in temperature occurred within the first half hour and moisture absorption by the hygroscopic fibers affected the temperature distribution. They also found that the water content accumulated with time and higher water content was found in the outer region than that in inner regions of the battings. This paper presents experimental investigations of water absorption of two natural low cost materials, coconut fiber and groundnut coquet. The samples of testing materials were bounded on their top by ice and the bottom surface was exposed to vapor with temperature close to $100^{\circ} \mathrm{C}$. The percentage of water accumulation due to vapor absorption was calculated for each layer.

\section{Experiments}

\subsection{Testing materials}

Coconut fibres and groundnut coquets, both natural low cost materials, are very abundant in nature. A coconut fibre is obtained by keeping the fruit envelope in water for 2 or 3 days. The fibres obtained can reach $30 \mathrm{~cm}$ in length with an average diameter of $0.3 \mathrm{~mm}$, fig. 1. Coconut fibre is characterized by its high percentage of lignin (40\%), which accounts for its stiffness when used for carpet manufacturing. Coconut fibres may be used as panels, bulk or rolls for wall insulation. Since it is flammable, it should be treated with boric salt to improve its resistance. 


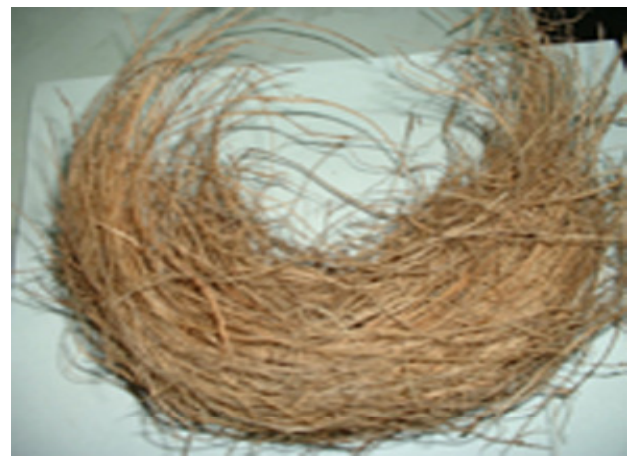

Figure 1: Coconut fibres.

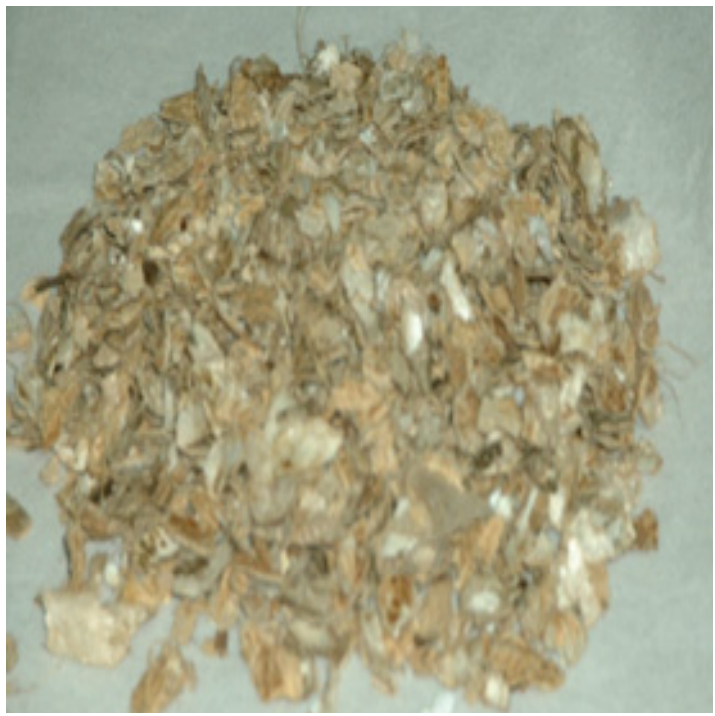

Figure 2: Groundnut coquets.

Groundnut coquettes are the outer jacket or shells of peanuts. Groundnut coquette has low density and long durability but must be protected from attack of insects. Very similar to wood, groundnut coquet may be treated to different dimensions, fig. 2, treated industrially to get chips for making assemblies such as plywood. The thermal conductivity of plywood varies with timber species, however an average value of $k=0.1154 \mathrm{~W} /\left(\mathrm{m}^{\circ} \mathrm{C}\right)$, (Edwin and William [3]) for softwood timbers is sufficiently accurate for determining the overall coefficient of heat transmission ( $U$ value) of a construction assembly. Thus, the decisive parameter for determining thermal conductivity of plywood is that of the wood species.

\subsection{Experimental procedure}

The experimental procedure was as follows, fig. 3: 
a) Produce an enclosure with 400x300x300 mm inside dimensions and $30 \mathrm{~mm}$ thick. The enclosure must be divided into two compartments by a metal plate $(300 \times 300 \mathrm{~mm})$ which will support on its top surface a $90 \mathrm{~mm}$ thick wall made with polyester. The metal plate must have several little holes at the center where the samples will be placed to allow vapor to flow through the testing materials. Also, the $90 \mathrm{~mm}$ thick polyester wall has a rectangular gap of 100x80 mm at the center. Make a gap of 100x100 mm to the bottom wall of the enclosure for vapor recirculation and, a second gap on the top wall to remove and put the samples.

b) Prepare the samples by making 3 layers with 100x80x30 mm dimensions, made with the materials to be tested. Cover the sample layers by wire mesh (wire diameter $0.071 \mathrm{~cm}$, strands per meter 315). The testing materials should be carefully packed to get the greatest density and reduce the amount of air. Weight the samples on an electronic balance and install them on the plate through the gap of the $90 \mathrm{~mm}$ thick polyester wall at the center of the enclosure. Connect the thermocouples to the layers horizontal surfaces and measure the temperature of each layer before testing.

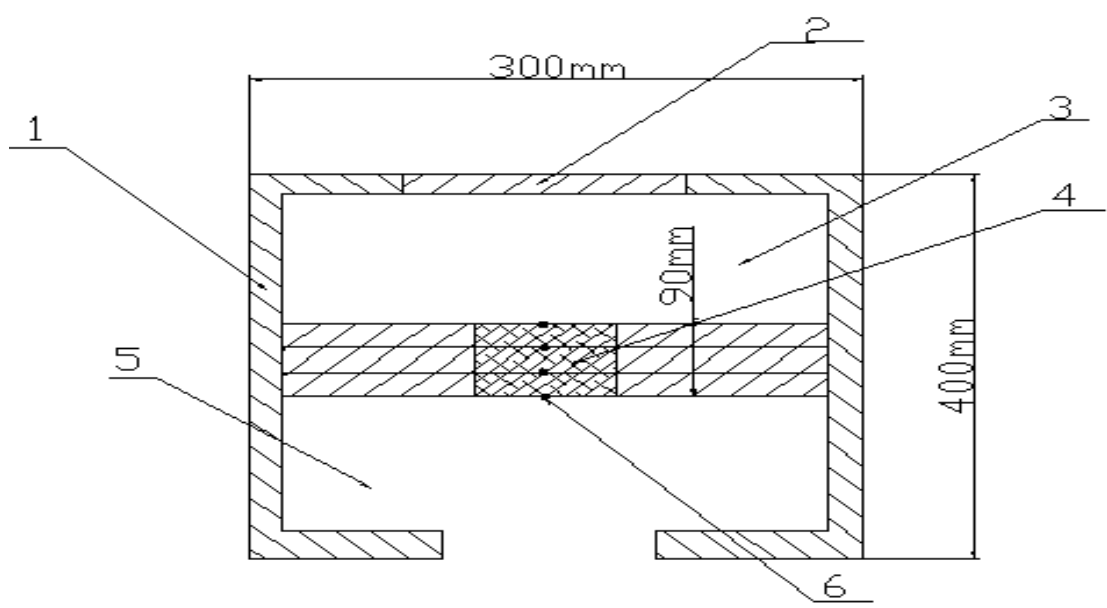

Figure 3: Experiment apparatus for water absorption analysis 1-Insulated box, 2-Gap to put and remove ice sack, 3- Space for ice, 4- Layers of testing materials, 5- Vapour recirculation, 6- Thermocouples connections.

c) Put ice cube in the upper compartment of the enclosure and supply water vapor to the other compartment below; so that the sandwich multi-layer (100x80x30 mm dimensions of each layer) of testing materials is bounded on its top by ice and the bottom surface is exposed to vapor. After 1 hour of experiment, remove the sample layers and weight them immediately; then calculate the percentage of water accumulation due to the absorption of vapor using eqn (1). Repeat the same procedure for $t=2 h, 3 h, 4 h, \ldots$ 


$$
w_{a c}=\frac{w_{i}-w_{o}}{w_{o}} 100_{\%}
$$

where $w_{i}$ is the layer weight after testing, $w_{o}$ the weight of the layer before testing, and $w_{a c}$ the increase of water content (in \%). The results are shown in fig. 5 and $6 ; x$ is the dimensionless distance from the layer one side of which is exposed to vapour.

$$
x=\frac{x_{i}}{d}
$$

where $x_{i}$ is distance from the surface exposed to vapour $\left(x_{i}=0\right.$ at the bottom and $x_{i}=90 \mathrm{~mm}$ at the top) and $d$ is the total thickness of the test sample.

The experimental uncertainty in weight is $0.1 \mathrm{~g}$; and thus, according to the procedure in Holman and Gajda [4] the uncertainty is about $6 \%$ in water content.

\subsection{Results and discussions}

A sandwich multi-layer (100x80x30 mm dimensions of each layer) of testing materials was bounded on its top by ice and the bottom surface was exposed to vapour with temperature close to $100^{\circ} \mathrm{C}$. Before testing, temperature at each location of thermocouple on the sample was about $20^{\circ} \mathrm{C}$. After one hour of testing, the temperature at the bottom reached a maximum of $98^{\circ} \mathrm{C}$ and remained almost invariable. At the top surface cooled by ice, the temperature first decreased slightly and then increased to about $30^{\circ} \mathrm{C}$ after one hour and finally reached $35^{\circ} \mathrm{C}$ after 4 hours. The percentage of water accumulation due to vapour absorption was calculated for each layer. Figures 5 and 6 present the measurements of the water absorption. Very clearly, the water content within both the coconut fibres and groundnut coquet increased with time and, the water content for the groundnut coquets was higher than that of the coconut fibres.

For the groundnut coquet sample made with low porosity and high solid fraction, the water content considerably increased for the layer exposed to water vapour within two hours; but, the increase in the top layer water content was faster fig. 4. The bottom layer water content accumulation increased rapidly after being decreased between 2 and 3 hours, this may be explained by the fact that when the amount of condensate within the top layer exceeds a certain value, the water liquid is expected to fall down to the bottom and increase the two first layers weights. During the third and the fourth hours, two motions of species clearly occurred: the flow of vapour from the bottom to the top layer and the flow of condensate water by gravity and capillary action from the top to the bottom layer. However, during the first and second hours there was almost no motion of water from the top to the bottom. The water was transferred mainly by diffusion through the sample which explains why the top layer water content 
216 Design and Nature III: Comparing Design in Nature with Science and Engineering

increased respectively with time within 3 hours where a few amount of condensate flowed down to the bottom.

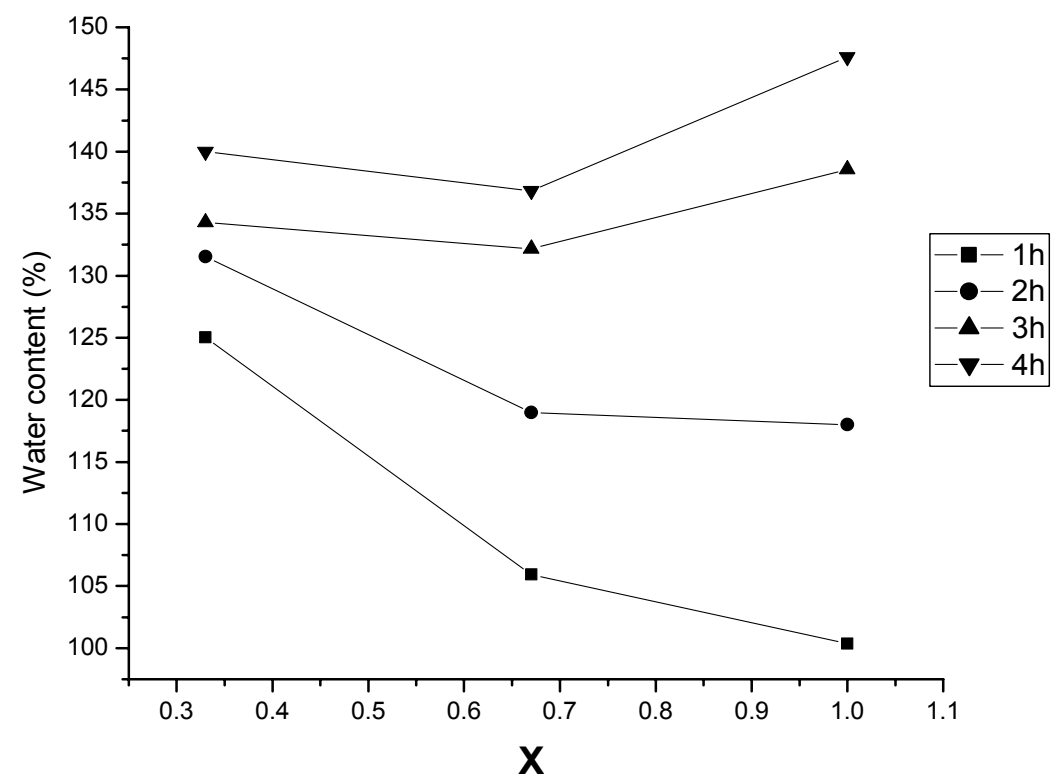

Figure 4: Water absorption for groundnut coquets.

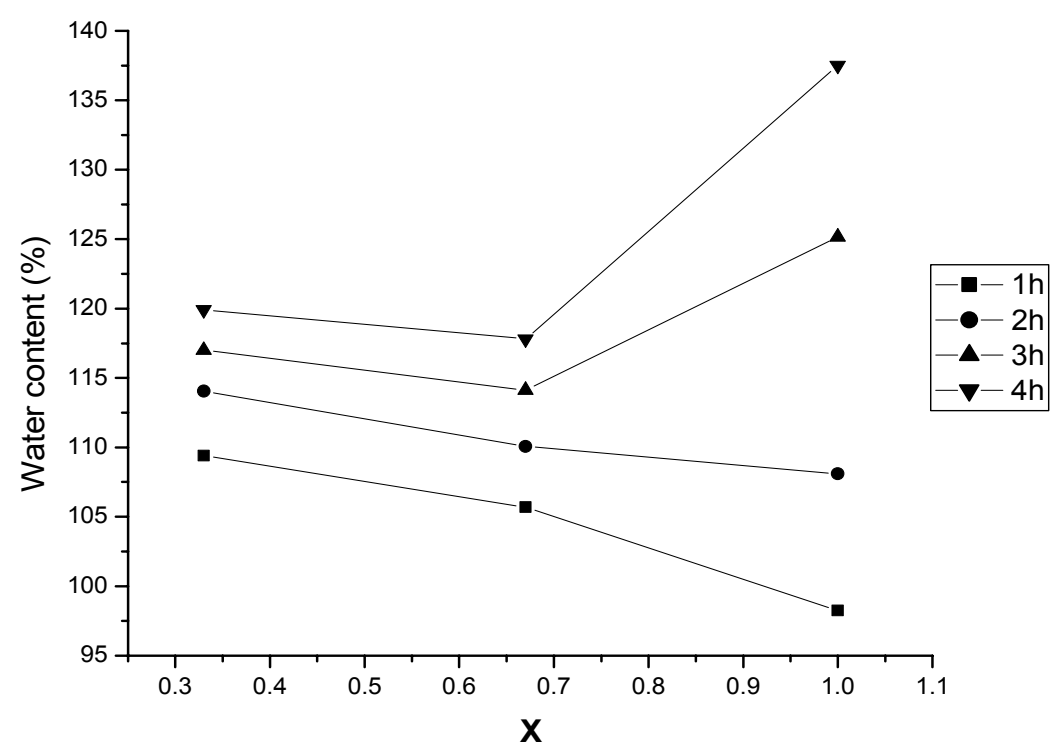

Figure 5: Water absorption for coconut fibres. 
The saturated vapour flowed easily into the coconut fibres sample due to the higher porosity or larger spaces between fibres. Therefore, the two first layers (from the surface exposed to vapour) were wetted almost uniformly during the whole experiment with higher water content for the first layer, fig. 5. The difference in water content was very small for the all three layers after 2 hours. The fast increasing for the top layer is due to the water accumulation at the top thanks to the impermeable plastic placed on the top surface. After 4 hours, the main amount of water was accumulated near the top and a few amount of the condensate water from the top layer flowed by capillary and gravity toward the bottom layer during the whole experiment. In this case, moisture transferred through void space is very important. The water accumulation and distribution within the testing material were due to the transport of warm and moist air through the material towards the cold surface. At some location within the insulation in the cold region, the insulation temperature would be below the saturation temperature and moisture would condense and soak the material. The water content distribution is also affected by the liquid motion on the fibres surfaces. When the amount of liquid condensate reaches some threshold, the liquid water overcomes the surface tension effects and moves to regions with lower water content. Finally, both the coconut fibres and the groundnut coquet at low temperatures and high humidity may absorb water.

The following equation of thermal conductivity assumes that the liquid is distributed in continuous layers parallel to the direction of heat flow, Choudhary et al. [5].

$$
k_{\text {eff }}=(1-\varepsilon) k_{s}+\varepsilon\left(s_{l} k_{l}+s_{g} k_{g}\right)
$$

where $\varepsilon$ is porosity; $k_{s}, k_{l}$ and $k_{g}$ are thermal conductivity of solid matter, liquid and gas, respectively; $s_{l}$ and $s_{g}$ are phase saturation for liquid and solid (volumetric fraction of the void space occupied by a specific phase).

Eqn (3) shows that the thermal conductivity of moist insulations depends considerably on the amount of the liquid it contains. As a result, since water is a better thermal medium, the thermal conductivity of the soaked insulation would increase and the insulating value of the material would be destroyed.

\section{Mathematical formulation}

A porous media heat and mass transfer model and the coordinate used are presented in fig. 6 which represents a multilayered sandwich made of the testing materials. One side of the sample is exposed to saturated water vapour at $\mathrm{z}=0$, where the temperature and the vapour concentration are To and wo, respectively. The opposite side is bounded by a plastic sheet and cooled by ice at $\mathrm{z}=\mathrm{d}$. When the saturated vapour flows through the porous media in the figure above, the following phenomenon may occur: diffusion of vapour through the pores in the materials (or fibres) toward the upper layer top surface; condensation on solid (cell) surface; diffusion of water within the cell wall; heat transfer due to 
temperature difference existing in the sample and condensation (condensation is accompanied with the release of heat latent which acts as a heat source in the heat transfer process); transport of liquid (condensate water) by gravity and capillary action from the top surface of the upper layer toward the first layer. Thus, three phases are present in the sample: the solid matrix, the liquid water and the gaseous phase composed of water vapour and air.

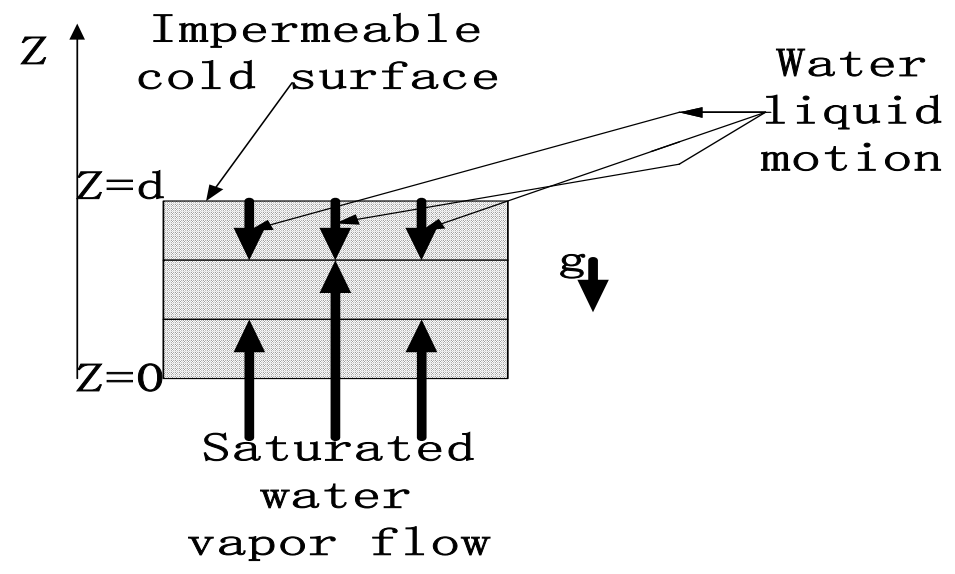

Figure 6: Analytical model and coordinate system for analysis.

The governing equations based on Whitaker's work, (Choudhary et al. [5], Whitaker [6]) may be obtained by applying the local volume averaging to the mass, momentum, and energy equation for individual phase. Assuming that the process is one dimensional and the following assumptions: the samples made of testing materials are homogeneous; convection in the solid matrix is neglected; the total gas-phase pressure in the sample is constant; dry air is stagnated in the sample; however the water vapour is mobile.

Energy equation

$$
\rho c_{p} \frac{\partial T}{\partial t}=\frac{\partial}{\partial z}\left(k_{e f f} \frac{\partial T}{\partial z}\right)-\dot{m} h_{f g}
$$

Water vapour diffusion equation

$$
\frac{\partial}{\partial t}\left(\varepsilon \rho_{g} s_{g} y_{v}\right)=\frac{\partial}{\partial z}\left(\varepsilon \rho_{g} s_{g} D \frac{\partial y_{v}}{\partial z}\right)+\dot{m}
$$

Water liquid transport equation 


$$
\frac{\partial}{\partial t}\left(\cos _{l}\right)=\frac{\partial}{\partial z}\left(D_{l} \frac{\partial s_{l}}{\partial z}\right)-\frac{\partial}{\partial z}\left(\xi K \frac{k_{r l}}{v_{l}} \rho_{l} g\right)-\dot{m}
$$

In Eqns (4), (5) and (6), the symbols $s_{g}$ and $s_{l}$ denote the phase saturation for gas and liquid, respectively. The phase saturation is defined as the volumetric fraction of the void space occupied by the corresponding phase. $c_{p}$ is specific heat $(\mathrm{J} / \mathrm{kg} \mathrm{K}), D$ binary diffusion coefficient for transport of water vapour $\left(\mathrm{m}^{2} / \mathrm{s}\right)$, $D_{l}$ liquid diffusivity $\left(\mathrm{m}^{2} / \mathrm{s}\right), g$ acceleration due to gravity $\left(\mathrm{m} / \mathrm{s}^{2}\right), h_{f g}$ latent heat $(\mathrm{J} / \mathrm{kg}), K$ absolute permeability $\left(\mathrm{m}^{2}\right), k_{\text {eff }}$ effective thermal conductivity $(\mathrm{W} / \mathrm{m} \mathrm{K})$,

$k_{r l}$ relative permeability, $m$ rate of phase change (negative for condensation) $\left(\mathrm{kg} / \mathrm{m}^{3} \mathrm{~s}\right), t$ time (s), $y_{v}$ mass fraction of water vapour in the gaseous mixture, $\varepsilon$ the porosity, $\rho$ the density $\left(\mathrm{kg} / \mathrm{m}^{3}\right), \xi$ hindrance function. The hindrance function varies from 0 (in the region that are fully saturated with liquid) to 1 (in the region that contains no water). The solutions of the eqns (4), (5) and (6) require more theoretical and numerical investigations. Further studies are needed to find an appropriate solution procedure to describe and solve numerically the thermodynamic and physical phenomenon of the figure 5 .

\section{Conclusion}

Experimental investigations are done to characterize the moisture absorption of two different natural materials (coconut fibres and groundnut coquets). A sandwich multi-layer (100x80x30 mm dimensions of each layer) of the testing materials was bounded on its top by ice and the bottom surface was exposed to vapour with temperature close to $100^{\circ} \mathrm{C}$. The percentage of water accumulation due to vapour absorption was calculated for each layer after a pre-set time of experiment $(1 \mathrm{~h}, 2 \mathrm{~h}, \ldots)$. The water content of the groundnut coquet was lower than that of the coconut fibres but for all the materials the water content increased with time. For the groundnut coquet sample, the water was transferred mainly by diffusion through the porous media; but in the coconut fibres sample with higher porosity, the water transferred by diffusion through void space was the most important. Actually, moisture absorption may occur when the materials are used under very low temperatures and high humidity. The thermal conductivity of moist insulation depends considerably on the amount of the liquid it contains; as a result, since water is a better thermal medium, the thermal conductivity of the soaked insulation would increase and the insulating value of the material would be destroyed. A thin plastic vapour barrier should be placed over the insulation to prevent moisture from entering the insulation.

\section{References}

[1] Conte, I., Peng, X., Christopher, D. M., Xie, J. \& Xu, J., Transport Properties of Biomass Materials as Thermal Insulator. Proc of the $7^{\text {th }}$ Asian Thermophysical Properties Conf., eds. B. X. Wang, pp. 63, 2004. 
220 Design and Nature III: Comparing Design in Nature with Science and Engineering

[2] Fan, J., Cheng, X. \& Chen, Y.-S., An experimental investigation of moisture absorption and condensation in fibrous insulations under low temperature. Experimental Thermal and Fluid Science, 27, pp. 723-729, 2002.

[3] Edwin, F. S. \& William C. T., Thermal Insulation Building Guide, Robert E. Krieger: Malabar, pp. 1-473, 1990.

[4] Holman, J. P. \& Gajda, W. J., Experimental Methods for Engineers $5^{\text {th }}$ edition, McGraw-Hill: United States, pp. 37-379, 1989.

[5] Choudhary, M. K., Karki, K. C. \& Patankar, S. V. Mathematical modeling of heat transfer, condensation, and capillary flow in porous insulation on a cold pipe, Int. Journal of Heat and Mass Transfer, 47, pp. 5629-5638, 2004.

[6] Whitaker, S., Theory and Applications of Transport in Porous Media: The Method of Volume Averaging, Kluwer Academic: USA, pp. 1-180, 1999. 